\title{
Changes in Thoracic Torsion in Adolescent Idiopathic Scoliosis Patients Within a 2-year Postoperative Period
}

\section{yonggang wang}

Lanzhou University Second Hospital

\section{Dongmin Wang}

Northwest Minzu University College of Medicine

\section{Xuewen Kang}

Lanzhou University Second Hospital

\section{Zhanjun Ma}

Lanzhou University Second Hospital

\section{Yingping $\mathrm{Ma}$}

Lanzhou University Second Hospital

\section{Xuchang Hu}

Lanzhou University Second Hospital

\section{Bing Ma}

Lanzhou University Second Hospital

\section{Yong Yang}

Lanzhou University Second Hospital

\section{Xuegang He}

Lanzhou University Second Hospital

\section{Shixiong Wang}

Lanzhou University Second Hospital

Bingren Gao ( $\sim 13462717 @ q q . c o m$ )

Lanzhou University Second Hospital

\section{Research article}

Keywords: adolescent idiopathic scoliosis, AIS, posterior surgical fusion, PSF, thoracic torsion

Posted Date: May 27th, 2021

DOI: https://doi.org/10.21203/rs.3.rs-531943/v1 
License: (c) (i) This work is licensed under a Creative Commons Attribution 4.0 International License. Read Full License 


\title{
Changes in thoracic torsion in adolescent idiopathic scoliosis patients within a 2-
} year postoperative period

Yonggang Wang ${ }^{1,2 \#}$, Dongmin Wang ${ }^{3 \#}$, Xuewen Kang ${ }^{2}$, Zhanjun $\mathrm{Ma}^{2}$, Yingping $\mathrm{Ma}^{2}$, Xuchang $\mathrm{Hu}^{2}$, Bing $\mathrm{Ma}^{2}$, Yong $\mathrm{Yang}^{2}$, Xuegang $\mathrm{He}^{2}$, Shixiong Wang ${ }^{1}$ and Bingren $\mathrm{Gao}^{1 *}$

\begin{abstract}
Background: Scoliosis can cause deformities of the rib cage. The three-dimensional (3D) shape of the rib-vertebral-sternal complex matches that of the thoracic cavity and is visualized as an elastic structural model that is approximately cubic in shape. This study was performed to evaluate the changes in thoracic torsion by measuring radiological parameters.
\end{abstract}

Methods: Forty-four patients with adolescent idiopathic scoliosis (AIS) with a main right thoracic curvature underwent posterior spinal fusion (PSF), and radiological parameters of the spine and thorax were evaluated.

Results: The correction of preoperative, immediately postoperative, and 2-year postoperative MT-Cobb angles were $64 \%$ and $66 \%$. The correction of T1-T12 heights were $10 \%$ and $12 \%$. The correction of RVAD was $59 \%$ immediately postoperatively and $52 \%$ at 2 year postoperatively. The correction of RH was 59\% immediately

*Correspondence: bingrengao@foxmail.com

\#Yonggang Wang and Dongmin Wang contributed equally to this work

1. Department of Cardiac Surgery, Lanzhou University Second Hospital, No. 82 Cuiyingmen Lanzhou 730030, Gansu province, China.

2. Department of Orthopedics, Lanzhou University Second Hospital, No. 82 Cuiyingmen Lanzhou 730030, Gansu province, China.

3. Medical College of Northwest Minzu University, No. 1 Northwest xincun, Lanzhou 730030, Gansu province, China 
postoperatively and $52 \%$ at 2 year postoperatively. The correction of AVB-R was $23 \%$ immediately postoperatively and $25 \%$ at 2 year postoperatively. From the above results, all radiological parameters were significantly different immediately and at 2 year postoperatively compared to preoperatively $(p<0.001)$. There were significant correlations between MT-Cobb angle and T1-T12 height $(p<0.001)$, RVAD $(p<$ $0.001)$, RH $(p<0.001)$, and AVB-R $(p<0.001)$.

Conclusion: Most cases of mild and moderate scoliosis surgery also includes the correction of thoracic torsion. PSF appears to be effective at correcting scoliosis, and the correction of thoracic torsion also plays an important role.

Keywords: adolescent idiopathic scoliosis; AIS; posterior surgical fusion; PSF; thoracic torsion

\section{Introduction}

Adolescent idiopathic scoliosis (AIS) is a three-dimensional (3D) spine deformity that comprises vertebral axial rotation, lateral frontal deviation, and sagittal intervertebral lordosis[1]. It can cause deformities of the rib cage, which is a primary concern for the patient and their family as it causes significant cosmetic issues, and severe scoliosis can also result in cardiopulmonary dysfunction.

In scoliosis, deformities in any plane do not develop in isolation, and the type and severity of the deformity may depend on the concurrent development of curvature, translation, and rotation in other planes[2,3]. Coronal deformity is the main problem in scoliosis. Due to secondary anatomical connections, this complex 3D thoracic deformity affects both the spine and the ribs[4]. The combined spine and rib cage deformity in scoliosis is best described as a thoracic deformity, and recent advances in imaging modalities have enabled better definition of 3D deformities of the thorax in scoliosis.

The rib-vertebrae-sternum complex, which was first introduced as a concept in $2007[2,5]$, exhibits a good 3D fit with the thoracic cavity and is usually visualized as an elastic structural model with an approximate cubic shape. Canavese et al. evaluated 
the effects of vertebral growth disorders on the development of the ribs, sternum, and lungs, which form part of the rib-vertebral-sternal complex[6,7].

Scoliosis can cause changes in costal joints and ribs. These distortions can produce a "convex" or "concave" half-thorax[8]. The deviation of the sternum with respect to the apical vertebra is the cause of lateral plane rotation deformity. If the thoracic deformity develops to a point where breathing, lung growth, or biomechanical movement is impaired, this condition is called thoracic insufficiency syndrome (TIS)[9].

During the growth of scoliosis patients, components of the rib-vertebral-sternal complex change together, raising questions about what changes will occur after scoliosis surgery. Timothy et al[10]. reviewed preoperative, immediately postoperative, and 2-year postoperative radiographic findings as well as pre- and post-operative computed tomography (CT) scans of 19 AIS patients with Lenke 1 curves. They reported that the thoracic torsion parameters exhibited moderate to good overall correlation with the main thoracic curve Cobb (MT-Cobb) angle, apical Perdriolle rotation, and apical CT rotation. These should be useful as clinical measures for assessing 3D deformity correction in plain radiographs, especially for the intraoperative evaluation of vertebral derotation and thoracic symmetry restoration. In comparison with $\mathrm{CT}$, anteroposterior (AP) radiography examination has limitations for evaluating thorax deformities caused by scoliosis. However, AP radiography is still used to evaluate and describe thorax deformities[11,12].

Elucidating the complex relationships between the spine, rib cage deformities, and pulmonary function can help improve clinical interventions for scoliosis treatment. Therefore, it is necessary to evaluate changes in lung function, taking into account not only the spinal curvature but also measurements of thoracic and rib deformities. At present, posterior spinal fusion (PSF) is the conventional surgical method for the treatment of scoliosis. Scoliosis is already known to cause changes in the thorax, but it is unclear what type of changes occur in the thoracic profile after scoliosis surgery. This 
study was performed to investigate changes in thoracic torsion in the postoperative period after scoliosis surgery.

\section{Materials and methods}

Data from 44 AIS patients treated with PSF between September 2015 and August 2017 at the Spinal Surgery Department of Lanzhou University Second Hospital (Lanzhou, China) were collected.

All patients were followed up for 2 year. Patient data included demographic data (age, sex, and body mass index $[\mathrm{BMI}])$, preoperative and postoperative standing AP radiographs, and standing lateral radiographs of the whole spine. The inclusion criteria were as follows: (1) AIS patient, (2) main thoracic curve $\geq 45^{\circ}$, (3) right thoracic scoliosis, (4) no other lung diseases, and (5) Lenke type 1, 2, or 3. We excluded patients with obesity due to its deleterious effects on lung volume and capacity in children and adolescents[13,14]. In addition to obese patients, we also excluded patients with respiratory failure and those with primary lung diseases that affect lung function, such as asthma and bronchitis.

All radiographic data were obtained using a Picture Archives and Communications System by two senior spine surgeons. The specific measurement methods and evaluation parameters used are outlined below[15-18] (Fig. 1, Fig. 2).

MT-Cobb angle: In a standing AP radiograph, the MT-Cobb angle is found between two intersecting lines, i.e., the upper endplate of the main thoracic curve upper vertebra and the lower endplate of the main thoracic curve lower vertebra[16,17] (Fig. 1).

Thoracic kyphosis (T5-T12 TK): Measured from the upper endplate of T5 to the lower endplate of T12[15,16] (Fig. 2).

Height of thoracic vertebrae 1-12 (T1-T12 height): The vertical distance between the upper endplate of T1 and the lower endplate of T12[10] (Fig. 1).

Rib-vertebra angle difference (RVAD): Measured according to the method described by Mehta[19,20], in which a perpendicular line is drawn to the middle of either the upper or lower border of the apical vertebra intersects another line drawn from the 
midpoint of the head of the rib to the midpoint of the neck of the rib, just medial to the region where the neck widens into the shaft of the rib. The rib line is extended medially to intersect the vertebral line to form the rib vertebrae angle (Fig. 1).

Apical vertebral body-rib ratio (AVB-R): The ratio of linear measurements from the lateral borders of the apical thoracic vertebrae to both left and right chest walls[10] (Fig. 1).

Apical rib hump prominence (RH): The linear distance between the left and right ribs at the rib deformity apex as measured in a lateral radiograph [10,21] (Fig. 2).

Thoracic transverse diameter (TD): The distance to the internal surfaces of the ribs above the costal attachments of the diaphragm[22] (Fig. 1).

Left and right thorax height: the distance from the apex of the thoracic cavity to the diaphragm dome in an AP radiograph[22] (Fig. 1).

Space available for lungs (SAL): With reference to an AP radiograph, the height of the hemithorax is defined as the distance from the middle of the most cephalic rib to the center of the hemidiaphragm. A ratio, expressed as a percentage, is derived by dividing the height of the concave hemithorax by the height of the convex hemithorax, defining the space available for the lungs[9].

Thoracic anteroposterior diameter: The distance from the chest wall to the anterior margin of the vertebrae at the diaphragm dome level or apical vertebra of kyphosis[22] (Fig. 2).

Thoracic sagittal longitudinal diameter: The distance from the apex of the thoracic cavity to the diaphragm dome in lateral view[22] (Fig. 2).

The correction rate associated with each parameter was calculated as follows:

$$
\% \text { Correction }=\frac{\mid \text { preoperative data }- \text { postoperative data } \mid}{\text { preoperative data }} \times 100 \%
$$

\section{Surgical methods}

The upper and lower fixed vertebrae were established according to Lenke's principle[16]. The joint capsule and surrounding soft tissue were released. Grade 1 and 
2 osteotomy were performed in the apical vertebral region[23]. The rod was bent according to the physiological bending angle of the sagittal plane. In addition, the rod was rotated, and the Cotrel-Dubousset method[24] was used for the orthopedic operation.

\section{Statistical analysis}

Statistical analysis was performed using SPSS statistical software (version 25; IBM, Armonk, NY, USA). Differences between preoperative, immediately postoperative, and 2-year postoperative indicators were evaluated using the paired $t$ test and Wilcoxon signed rank test. Similarly, where appropriate, Pearson's correlation coefficient or Spearman's rank order correlation was used to assess correlations among the parameters. Qualitatively, we considered a correlation coefficient of $0.0-0.24$ to indicate no to poor correlation, $0.25-0.49$ low to moderate correlation, $0.50-0.75$ moderate to good correlation, and $0.76-1.0$ good to excellent correlation[25,26]. In all analyses, $p<0.05$ was taken to indicate significance.

\section{Results}

\section{Assessment of demographic and spinal deformity characteristics}

Patient demographic data such as sex, age, standing height and weight, and BMI are summarized in Table 1. In all AIS patients, radiological characteristics indicated a right main thoracic curve. The most common curve pattern was Lenke type 1, which was found in 27 patients $(61.3 \%)$. Lenke type 2 and Lenke type 3 were the second- and third-most common curve patterns, occurring in $10(22.7 \%)$ and five $(11.4 \%)$ of the patients, respectively. The apical vertebra of the main thoracic curve corresponded to $\mathrm{T} 7$ in four cases, T8 in 11 cases, T9 in 24 cases, and T10 in five cases.

\section{Surgical treatment and follow-up}

As noted, all patients underwent selective PSF of the main thoracic curve with a pedicle screw and double 5.5-mm rod. The segments that underwent fusion were selected for 
each patient according to the Lenke classification[16,27,28]. An average of 9.98 levels were fused per patient (range, 7-16). Postoperative follow-up averaged 14 months (range, 11-17 months). Clinical outcomes were not assessed in this study. However, there were no neurological deficits or other major complications, implant failures, or radiographic pseudarthrosis in the study group.

\section{Spinal assessments}

The average preoperative, immediately postoperative, and 2-year postoperative MTCobb angles were $58.70^{\circ} \pm 1.86^{\circ}\left(\right.$ range, $\left.45^{\circ}-94^{\circ}\right), 21.76^{\circ} \pm 1.74^{\circ}\left(\right.$ range, $5^{\circ}-47^{\circ}, 64 \%$ correction), and $20.56^{\circ} \pm 1.62^{\circ}$ (range, $5^{\circ}-45^{\circ}, 66 \%$ correction), respectively (Table 2). Both the immediately and 2-year postoperative MT-Cobb angles were significantly different from the preoperative value $(p<0.001)$.

The average preoperative, immediately postoperative, and 2-year postoperative TK (T5-T12) was $32.72^{\circ} \pm 2.66^{\circ}, 20.55^{\circ} \pm 1.60^{\circ}$, and $19.77^{\circ} \pm 1.36^{\circ}$, respectively (Table 2). Both the immediately and 2-year postoperative TK (T5-T12) was significantly different from the preoperative value $(p<0.001)$.

The average preoperative, immediately postoperative, and 2-year postoperative T1T12 heights were $234.16 \pm 3.85 \mathrm{~mm}$ (range, 172-295 mm), $257.07 \pm 4.33 \mathrm{~mm}$ (range, 180-326 mm), and 260.86 $\pm 4.17 \mathrm{~mm}$ (range, 193-332 mm), respectively (Table 2). The correction rates immediately and at 2 year postoperatively were $10 \%$ and $12 \%$, respectively. The immediately and 2-year postoperative T1-T12 heights were significantly different from the preoperative value $(p<0.001)$.

\section{Thoracic torsion assessments}

After PSF for scoliosis, the RVAD was reduced from $33.70^{\circ} \pm 2.76^{\circ}$ preoperatively to $14.61^{\circ} \pm 1.95^{\circ}$ immediately postoperatively and $18.43^{\circ} \pm 2.17^{\circ}$ at 2 year postoperatively, representing correction rates of 59\% and 52\%, respectively (Table 3). The RVADs immediately postoperatively and at 2 year postoperatively were significantly different from the preoperative value $(p<0.001)$. 
The mean RH was $22.04 \pm 1.34 \mathrm{~mm}$ preoperatively and improved to $9.66 \pm 1.02 \mathrm{~mm}$ immediately postoperatively and $6.09 \pm 1.14 \mathrm{~mm}$ at 2 year postoperatively, representing correction rates of $58 \%$ and $76 \%$, respectively (Table 3). The mean RHs immediately and at 2 year postoperatively were significantly different from the preoperative value $(p<0.001)$. Moreover, the value at 2 year postoperatively was also significantly different from value immediately postoperatively $(p<0.05)$.

After orthopedic treatment for scoliosis, AVB-R improved from $2.09 \pm 0.11$ preoperatively to $1.61 \pm 0.09$ immediately postoperatively and $1.52 \pm 0.55$ at 2 year postoperatively, representing correction rates of 23\% and 25\%, respectively (Table 3). The values immediately and at 2 year postoperatively were significantly different from the preoperative value $(p<0.001)$.

SAL improved significantly from $1.00 \pm 0.01$ preoperatively to $1.08 \pm 0.01(p<0.001)$ immediately postoperatively and $1.04 \pm 0.01(p<0.05)$ at 2 year postoperatively (Table 3).

There were no significant changes in TD, thoracic anteroposterior diameter (diaphragm level), thoracic sagittal longitudinal diameter, left and right thorax height, as shown in

\section{Table 3.}

\section{Correlation analysis}

The relationships between MT-Cobb angle and RVAD, RH, AVB-R, and T1-T12 height are shown in Table 4. MT-Cobb angle was significantly correlated with T1-T12 height $\left(\mathrm{R}^{2}=0.524, p<0.001\right)$ (Fig. 3), $\mathrm{RVAD}\left(\mathrm{R}^{2}=0.320, p<0.001\right)$ (Fig. 4), $\mathrm{RH}\left(\mathrm{R}^{2}\right.$ $=0.497, p<0.001)\left(\right.$ Fig. 5), and AVB-R $\left(\mathrm{R}^{2}=0.356, p<0.001\right)$ (Fig. 6).

\section{Discussion}

As the spinal deformity progresses, not only is spinal growth affected but the size and shape of the rib cage are also modified. Therefore, scoliosis is a 3D spinal deformity. With the application of modern instrument technology, the 3D deformity in AIS can be corrected. That is, current technology can resolve the coronal, sagittal, and axial 
deformities associated with scoliosis. Given the tools currently available, the main purpose of the present study was to determine whether $3 \mathrm{D}$ thoracic deformities observed in AIS can be corrected.

CT and MRI can provide the level of detail needed to assess the out-of-plane rotation of the spine and changes in chest volume that often accompany scoliosis. AP radiography is currently the gold standard for evaluating scoliosis and is a commonly used imaging modality due to the low level of radiation exposure, making it useful for longitudinal observation. Although it is sufficient for assessing the curvature of the spine in the coronal and sagittal planes, AP radiography is insufficient for describing vertebral rotation and thoracic distortion[10,28].

In the present study, MT-Cobb angles immediately and at 2 year postoperatively were significantly smaller than the preoperative angle (Table 2), reflecting obvious correction, and there was no significant change at 2 year postoperatively compared to immediately postoperatively. TK (T5-T12) immediately and at 2 year postoperatively was significantly less than that preoperatively (Table 2), which was mainly related to the bending of the rod and not to the correction of the MT-Cobb angle. After correction, TK (T5-T12) values fell within the normal range.

Watanabe et al.[29] evaluated the correlation between curvature correction and increased spinal height in AIS. The spinal height in the anterior spinal fusion group and the PSF group increased significantly postoperatively. The correction of the MT-Cobb angle was strongly correlated with the increase in T1-L5 height, especially in the PSF group. In our study of spinal height changes, we found that the T1-T12 height increased significantly immediately and at 2 year postoperatively compared to preoperatively (Table 2). There was a significant correlation between the correction rate of the MTCobb angle and the rate of increase in T1-T12 height (Table 4, Fig. 3), consistent with the findings of Watanabe et al.[29]. The T1-T12 height represents the height of the thorax, and an increase in this height indicates improvement in the thorax. 
The RVAD (also known as the Mehta angle) represents the asymmetry of the apical ribs in traditional radiographs. It was introduced as a prognostic factor for the severity of early-onset scoliosis and later applied to other types of scoliosis[30]. There is a strong relationship between the MT-Cobb angle, curve bending, the Mehta angle, and apical vertebral rotation. Together with the clinical Adams test, these are the most important radiographic torsion-related parameters in the evaluation of 2D scoliosis[11]. Therefore, there are questions regarding changes in the RVAD due to correction of the MT-Cobb angle. The results of the present study showed that the correction rate of MTCobb well-correlated with changes in the RVAD (Table 4). The RVAD immediately and at 2 year postoperatively differed significantly from the preoperative value (Table 3).

The AVB-R is related to apical vertebral translation and is used to evaluate the correction of thoracic and lumbar spine deformities as well as scoliosis. However, in addition to assessing displacement in terms of thoracic apical vertebral translation, AVB-R can also be used to assess the overall deformity of the thoracic spine and ribs $[10,27,31]$. The AVB-R changed obviously immediately postoperatively, indicating that the apical vertebra exhibited good midline deviation and the coronal correction was good. During follow-up, the fusion outcomes were stable, and no deviation was observed (Table 3). The AVB-R correction rate was well-correlated with that of the MT-Cobb angle (Table 4).

Trunk deformities include the rotation of the vertebral body and deformities of the back of the vertebral body and ribs. Previously, the main method of treatment for rib humps was thoracoplasty, but with the emergence of pedicle screws and the improvement of orthopedic techniques, surgical corrections of vertebral rotation deformities have achieved significant results. At the same time, secondary rib humps are also corrected. For mild scoliosis, the improved methods have the same outcomes as thoracoplasty, so thoracoplasty is now rarely employed[32]. The natural improvement rate of rib humps in patients without thoracoplasty is estimated to be $37 \%$, whereas that in patients 
undergoing thoracoplasty is $40 \%$. Although this difference is statistically significant, there is no significant difference in terms of clinical outcome[33]. Direct vertebral body rotation and vertebral coplanar alignment techniques allow more effective vertebral body rotation and rib hump correction[34]. Patients who did not undergo thoracoplasty exhibited a spontaneous improvement of the clinical rib hump of $37 \%$. Similar results were obtained in the present study. The RH was well-correlated with MT-Cobb angle correction rate (Table 4), and preoperative and postoperative values were significantly different (Table 3). Rib humps were also significantly reduced.

Spinal deformity is one cause of TIS, defined as the inability of the chest to support breathing or lung growth. Spinal deformity leads to thoracic deformity with a loss of volume and function[9,35,36]. The TD at the apical vertebra, TD at the diaphragm level, thoracic anteroposterior diameter (diaphragm level), thoracic sagittal longitudinal diameter, left SAL, and right SAL, are good indicators for evaluating thoracic deformity and TIS[22,37-39]. They are used as indicators of thoracic growth and development, and are mostly used to evaluate the differences in height and asymmetry of the rib cage caused by deformity of the left and right rib cages. With the exception of SAL, they were not significantly different among the three time points examined (preoperatively, immediately postoperatively, and at 2 year postoperatively). SAL improved significantly from $1.00 \pm 0.01$ preoperatively to $1.08 \pm 0.01 \quad(p<0.001)$ immediately postoperatively and $1.04 \pm 0.01(p<0.05)$ at 2 year postoperatively.

This study had some limitations. When the radiographs were taken, there was no clear indication of when each patient exhaled and inhaled, which might have led differences in chest expansion and location of the lower lung boundary. Therefore, there were some errors in the measurement of the transverse and sagittal chest diameters. In addition to the influence of thoracic volume on lung function, the functional status of other organs, such as the lungs and respiratory muscles, could also affect the results. There have been no studies of the rotation of the apical vertebra. Because of the influence of the postoperative instrument, the rotation of the vertebral body cannot be measured 
accurately, and no CT was performed in postoperative examinations because of the high dose of radiation required.

\section{Conclusion}

Most cases of mild and moderate scoliosis involve thoracic deformity, so correction of thoracic torsion is part of scoliosis surgery. Previously, thoracoplasty was performed to treat thoracic torsion, especially to improve the RH. Our findings confirm that there is a significant correlation between thoracic torsion and MT-Cobb angle. PSF can effectively correct scoliosis, and the correction of thoracic torsion also plays a role. This study showed that the correction of thoracic torsion can be evaluated based on the appearance of the thorax or radiography. Parameter correction rates were strong correlated, especially between those of the MT-Cobb angle and RH, AVB-R, and RVAD. We evaluated the correction of thoracic torsion by estimating the MT-Cobb correction rate. There were no significant changes in the transverse and sagittal diameters of the thorax after spinal correction, but the T1-T12 height improved significantly after surgery. In conclusion, with the PSF surgical technique, a greater MT-Cobb angle correction rate is associated with increased RH, T11-T12 height, AVB-R, and RVAD correction rates, and these correction rates are positively correlated.

\section{Abbreviations}

AIS: Adolescent idiopathic scoliosis; 3D: The three-dimensional; PSF: Posterior spinal fusion; MT-Cobb:Main thoracic curve Cobb's angle ; T1-T12 height: height of thoracic vertebrae 1 to 12 ; RVA: Rib vertebra angle ; AVB-R: Apical vertebral bodyrib ratio; TK:Thoracic kyphosis; RH: Apical rib hump prominence

MT-Cobb: MT-Cobb angle; T1-T12: Thoracic vertebrae 1-12; TK: Thoracic kyphosis; RVAD: Rib-vertebra angle difference; AVB-R ratio: Main thoracic curve apical 
vertebral body-to-rib ratio; RH: Apical rib hump prominence; TD: Thoracic transverse diameter; SAL: Space available for lungs; CT: computed tomography scans

\section{Ethics approval and consent to participate}

Ethical approval was obtained by Lanzhou University Second Hospital Medical Ethics Committee Review Form. Approval reference if relevant. We had all the necessary consent from any of the patients involved in the study, including consent to participate in the study where appropriate.

\section{Consent for publication}

Not applicable

\section{Availability of data and materials}

All data were true and effective, and all patients were hospitalized in our hospital.

\section{Competing interests}

The authors declare that they have no competing interests.

\section{Funding}

This work was financially supported by the Chinese Medicine Administration Research Project of Gansu province (GZK-2019-46) and Cuiying Technology Innovation Project of Lanzhou University Second Hospital (CY2019-MS10 and CY2020-MS20). The author is grateful for the enthusiastic efforts of all the people who have done this work.

\section{Authors' contributions}

YW and BG contributed in the conception and design, analysis and interpretation, and writing of the manuscript. DW, ZM, YM, XH, YY and SW contributed to the data collection and material support. $\mathrm{XH}$ and $\mathrm{BM}$ contributed to the critical revision of the manuscript and supervision. All authors read and approved the final manuscript.

\section{Acknowledgements}

The author is grateful for the enthusiastic efforts of all the people who have done this work.

\section{References}

1. Courvoisier A, Drevelle X, Dubousset J, Skalli W. Transverse plane 3D analysis of mild scoliosis. Eur Spine J 
$2013 ; 22(11): 2427-32$.

2. Shin JH, Wang S, Yao Q, Wood KB, Li G. Investigation of coupled bending of the lumbar spine during dynamic axial rotation of the body. Eur Spine J 2013;22(12) :2671-7.

3. Nault ML, Mac-Thiong JM, Roy-Beaudry M, deGuise J, Labelle H, Parent S. Three-dimensional spine parameters can differentiate between progressive and nonprogressive patients with AIS at the initial visit: a retrospective analysis. J Pediatr Orthop $2013 ; 33(6): 618-23$.

4. Illes TS, Lavaste F, Dubousset JF. The third dimension of scoliosis: The forgotten axial plane. Orthop Traumatol Surg Res $2019 ; 105(2): 351-59$.

5. Canavese F, Dimeglio A, Volpatti D, Stebel M, Daures JP, Canavese B, et al. Dorsal arthrodesis of thoracic spine and effects on thorax growth in prepubertal New Zealand white rabbits. Spine (Phila Pa 1976) 2007;32(16):E443-50.

6. Canavese F, Dimeglio A, Stebel M, Galeotti M, Canavese B, Cavalli F. Thoracic cage plasticity in prepubertal New Zealand white rabbits submitted to T1-T12 dorsal arthrodesis: computed tomography evaluation, echocardiographic assessment and cardiopulmonary measurements. Eur Spine J 2013;22(5) :1101-12.

7. Dimeglio A, Canavese F. The growing spine: how spinal deformities influence normal spine and thoracic cage growth. Eur Spine J $2012 ; 21(1): 64-70$.

8. Grivas TB, Vasiliadis ES, Mihas C, Savvidou 0. The effect of growth on the correlation between the spinal and rib cage deformity: implications on idiopathic scoliosis pathogenesis. Scoliosis $2007 ; 2: 11$.

9. Campbel1 RM, Jr., Smith MD, Mayes TC, Mangos JA, Willey-Courand DB, Kose N, et al. The characteristics of thoracic insufficiency syndrome associated with fused ribs and congenital scoliosis. J Bone Joint Surg Am 2003;85(3) :399-408.

10. Kuklo TR, Potter BK, Lenke LG. Vertebral rotation and thoracic 
torsion in adolescent idiopathic scoliosis: what is the best radiographic correlate? J Spinal Disord Tech 2005;18(2) :139-47.

11. Pizones J, Zuniga L, Sanchez-Mariscal F, Izquierdo E. Relationship between the different torsion-related thoracic deformity parameters of adolescent idiopathic scoliosis. Eur J Orthop Surg Traumatol 2016;26(7):763-9.

12. Grivas TB, Dangas S, Polyzois BD, Samelis P. The Double Rib Contour Sign (DRCS) in lateral spinal radiographs: aetiologic implications for scoliosis. Stud Health Technol Inform $2002 ; 88: 38-43$.

13. Winck AD, Heinzmann-Filho JP, Soares RB, da Silva JS, Woszezenki CT, Zanatta LB. Effects of obesity on lung volume and capacity in children and adolescents: a systematic review. Rev Paul Pediatr 2016;34(4):510-17.

14. Jones RL, Nzekwu MM. The effects of body mass index on lung volumes. Chest $2006 ; 130(3): 827-33$.

15. Winter RB, Lonstein JE, Denis F. Sagittal spinal alignment: the true measurement, norms, and description of correction for thoracic kyphosis. J Spinal Disord Tech 2009;22(5):311-4.

16. Zhang $\mathrm{H}$ SD, Richards BS. Principles of Surgical Plan for Adolescent Idiopathic Scoliosis. 1st ed. Beijing China: People’ s Health Publishing House; 2015.

17. Malfair D, Flemming AK, Dvorak MF, Munk PL, Vertinsky AT, Heran MK, et al. Radiographic evaluation of scoliosis: review. AJR Am J Roentgenol 2010;194(3 Supp1):S8-22.

18. Harris JA, Mayer OH, Shah SA, Campbe11 RM, Jr., Balasubramanian S. A comprehensive review of thoracic deformity parameters in scoliosis. Eur Spine J 2014;23(12) :2594-602.

19. Mehta MH. The rib-vertebra angle in the early diagnosis between resolving and progressive infantile scoliosis. J Bone Joint Surg Br $1972 ; 54(2): 230-43$.

20. Tysklind RG, Myung K, Gantsoudes G, Kishan S, Turner C, Lee S, et al. Intraobserver and Interobserver Measurement Variability 
of the Rib-Vertebral Angle Difference. J Pediatr Orthop $2018 ; 38(9): 455-58$.

21. Thulbourne T, Gillespie R. The rib hump in idiopathic scoliosis. Measurement, analysis and response to treatment. J Bone Joint Surg Br 1976;58(1):64-71.

22. Xue X, Shen J, Zhang J, Zhao H, Li S, Wang Y, et al. An analysis of thoracic cage deformities and pulmonary function tests in congenital scoliosis. Eur Spine J 2015;24(7):1415-21.

23. Schwab F, Blondel B, Chay E, Demakakos J, Lenke L, Tropiano P, et al. The comprehensive anatomical spinal osteotomy classification. Neurosurgery 2015;76 Supp1 1:S33-41; discussion S41.

24. Gioia G, Dubousset J, M'Rabet A. Treatment of adolescent scoliosis by the Cotre1-Dubousset method. Ital J Orthop Traumatol $1987 ; 13(4): 509-16$.

25. Sundmacher L, Gotz N, Vogt V. [Statistical methods for research on regional health-care services]. Bundesgesundheitsblatt Gesundheitsforschung Gesundheitsschutz 2014;57(2):174-9.

26. Banting J, Meriano T. Basic Biostatistics and Clinical Medicine. J Spec Oper Med;17(1):76.

27. Lenke LG, Betz RR, Harms J, Bridwell KH, Clements DH, Lowe TG, et al. Adolescent idiopathic scoliosis: a new classification to determine extent of spinal arthrodesis. J Bone Joint Surg Am $2001 ; 83(8): 1169-81$.

28. Aaro S, Dahlborn M. Estimation of vertebral rotation and the spinal and rib cage deformity in scoliosis by computer tomography. Spine (Phila Pa 1976) 1981;6(5):460-7.

29. Watanabe K, Hosogane N, Kawakami N, Tsuji T, Toyama Y, Chiba K, et al. Increase in spinal longitudinal length by correction surgery for adolescent idiopathic scoliosis. Eur Spine J $2012 ; 21(10): 1920-5$.

30. Brink RC, Schlosser TPC, van Stralen M, Vincken KL, Kruyt MC, Chu WCW, et al. What Is the Actual 3D Representation of the Rib 
Vertebra Angle Difference (Mehta Angle)? Spine (Phila Pa 1976) 2018;43 (2) :E92-E97.

31. Kuklo TR, Potter BK, Polly DW, Jr., Lenke LG. Monaxial versus multiaxial thoracic pedicle screws in the correction of adolescent idiopathic scoliosis. Spine (Phila Pa 1976) $2005 ; 30(18): 2113-20$.

32. Lykissas MG, Sharma V, V VJ, Crawford AH. Assessment of rib hump deformity correction in adolescent idiopathic scoliosis with or without costoplasty using the double rib contour sign. J Spinal Disord Tech 2015;28(4) :134-9.

33. Min K, Waelchli B, Hahn F. Primary thoracoplasty and pedicle screw instrumentation in thoracic idiopathic scoliosis. Eur Spine J 2005;14(8):777-82.

34. Rushton PR, Grevitt MP. Do vertebral derotation techniques offer better outcomes compared to traditional methods in the surgical treatment of adolescent idiopathic scoliosis? Eur Spine J $2014 ; 23(6): 1166-76$.

35. Mayer 0, Campbel1 R, Cahill P, Redding G. Thoracic Insufficiency Syndrome. Curr Probl Pediatr Adolesc Health Care 2016;46(3) :7297.

36. Campbell RM, Jr., Smith MD. Thoracic insufficiency syndrome and exotic scoliosis. J Bone Joint Surg Am 2007;89 Supp1 1:108-22.

37. Suwatanapongched T, Gierada DS, Slone RM, Pilgram TK, Tuteur PG. Variation in diaphragm position and shape in adults with normal pulmonary function. Chest 2003;123(6):2019-27.

38. Obikili EN, Okoye IJ. Transverse thoracic diameter in frontal chest radiographs of an adult Nigerian population. West Afr J Med $2006 ; 25(3): 186-9$.

39. Campbel1 RM, Jr., Smith MD, Mayes TC, Mangos JA, Willey-Courand DB, Kose N, et al. The effect of opening wedge thoracostomy on thoracic insufficiency syndrome associated with fused ribs and congenital scoliosis. J Bone Joint Surg Am 2004;86(8):1659-74. 
Figures

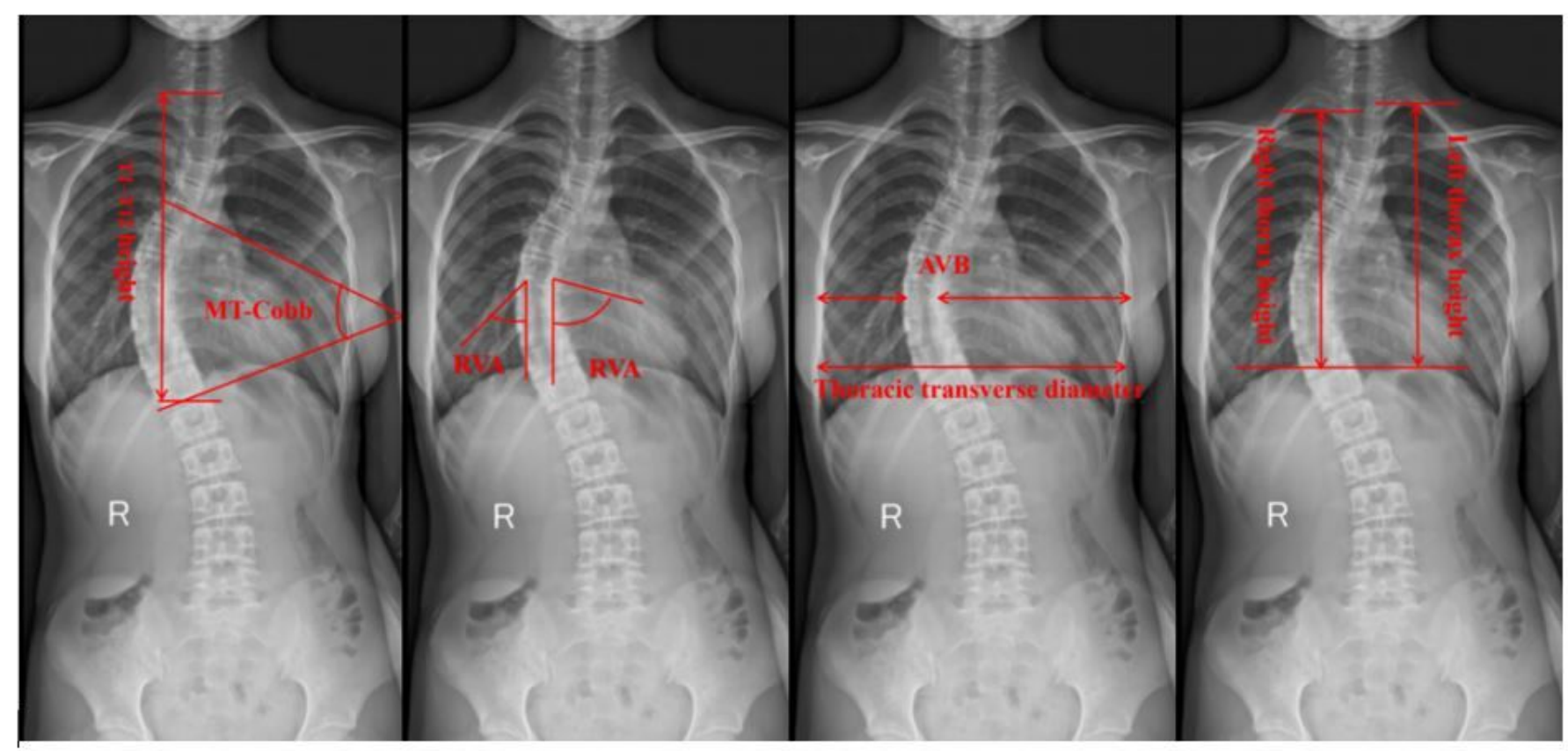

\section{Figure 1}

Radiographic parameters of the spine and thoracic coronal plane (MT-Cobb:Main thoracic curve Cobb's angle ; T1-T12 height: height of thoracic vertebrae 1 to 12; RVA: Rib vertebra angle ; AVB-R: Apical vertebral body-rib ratio). 


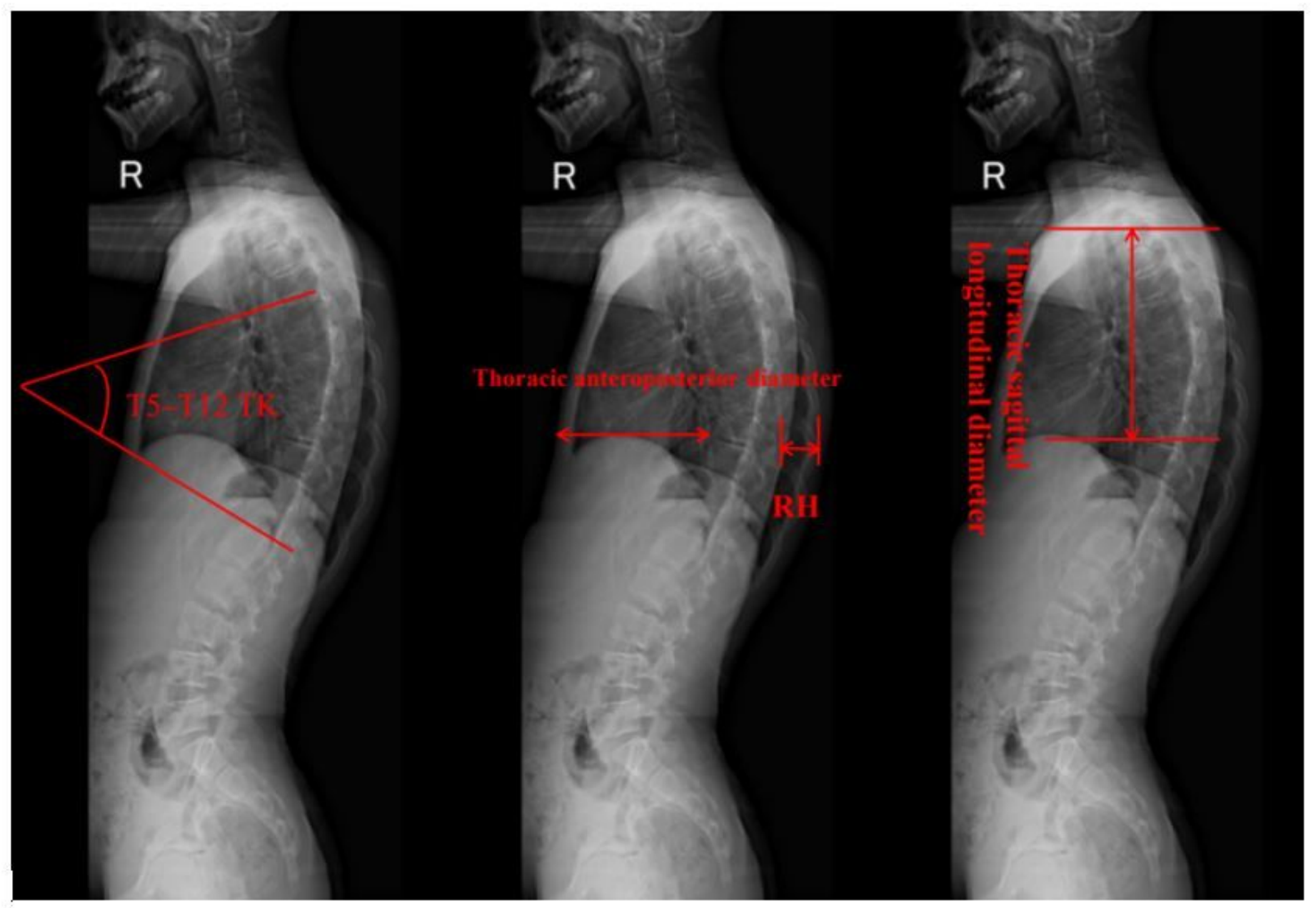

Figure 2

Radiographic parameters of the spine and thoracic sagittal plane (T5-T12 TK:Thoracic kyphosis; RH: Apical rib hump prominence). 


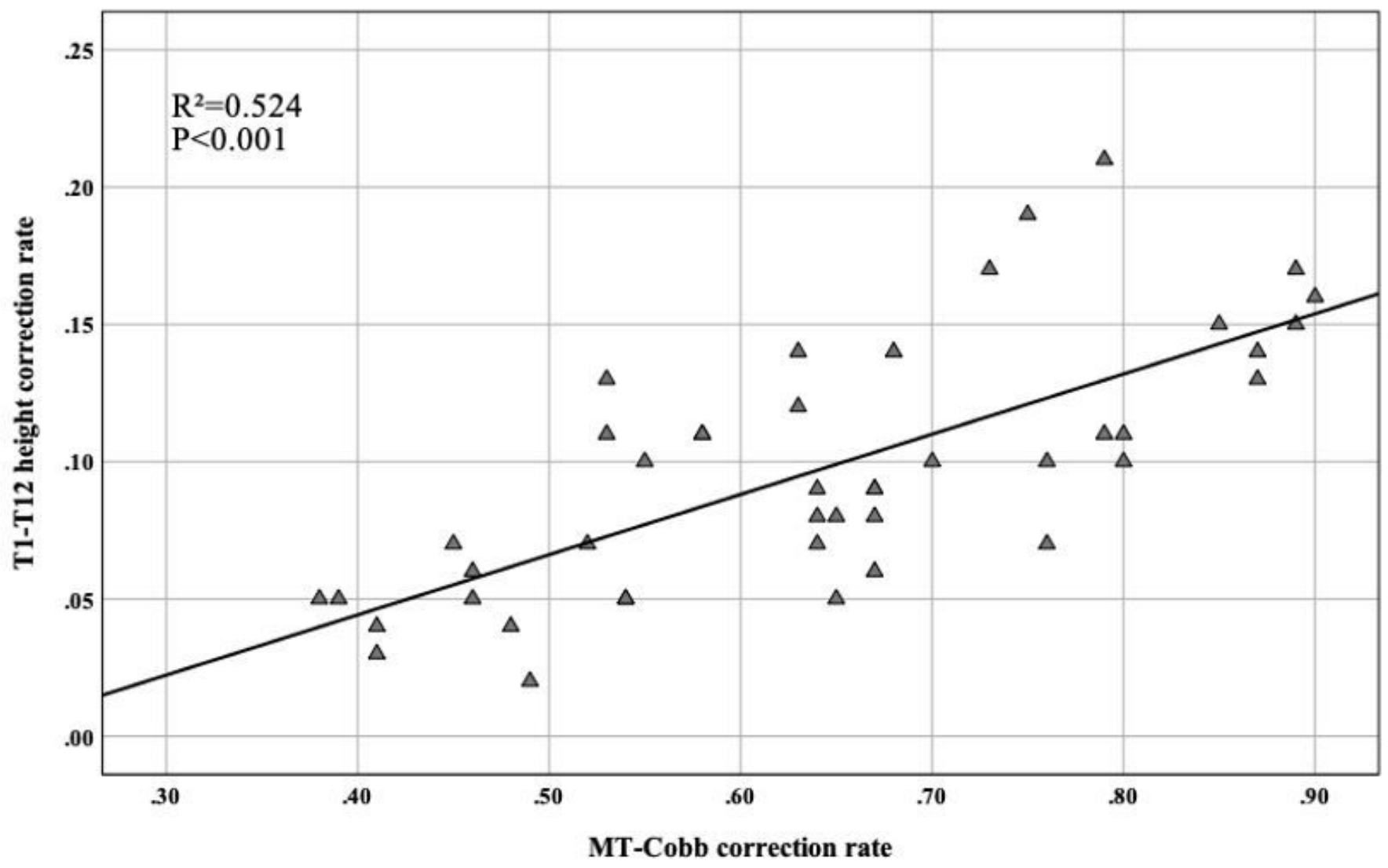

Figure 3

Correlation between main thoracic curve (MT)-Cobb angle and height of thoracic vertebrae 1-12 (T1T12 height) correction rates 


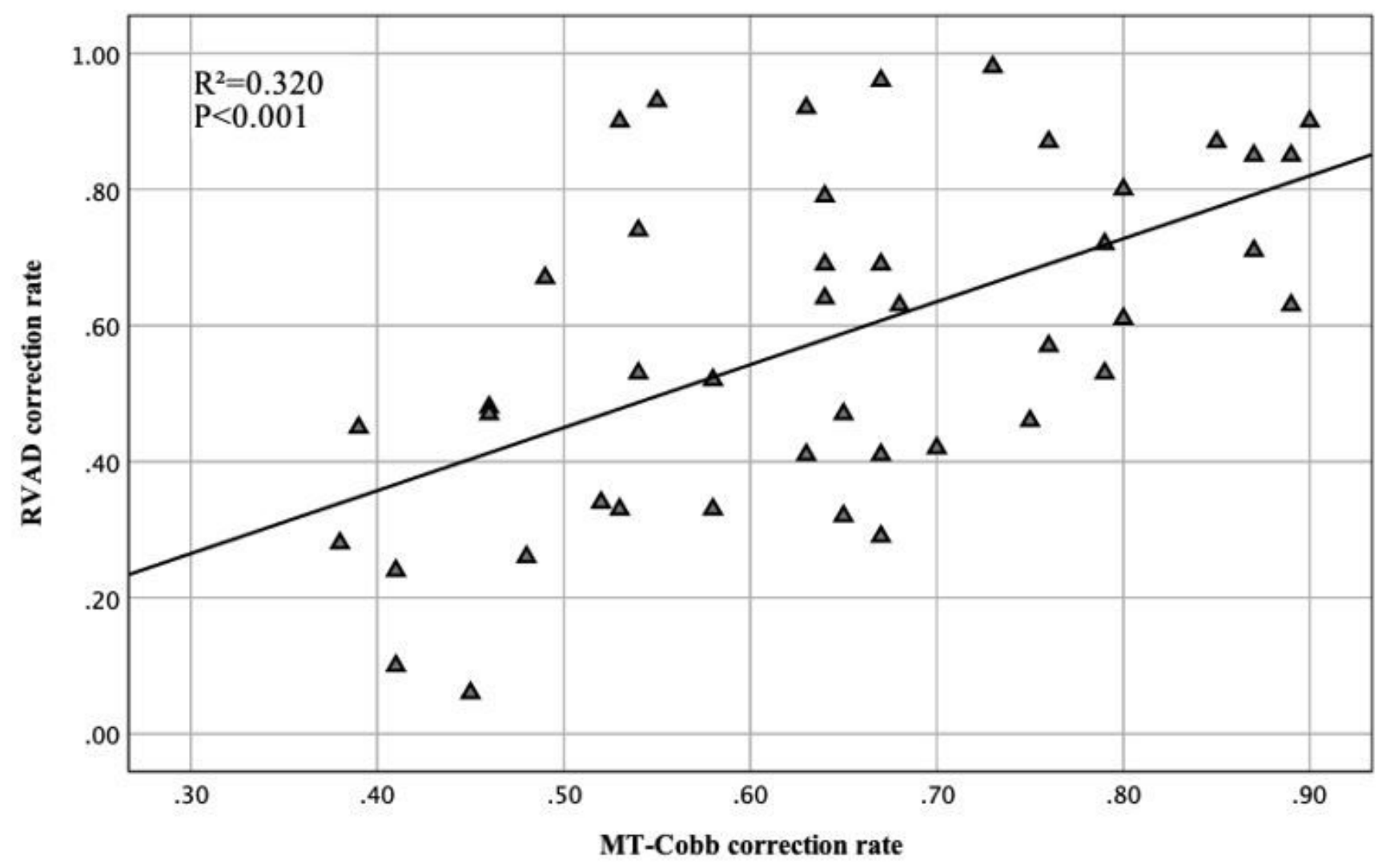

Figure 4

Correlation between MT-Cobb angle and rib-vertebra angle difference (RVAD) correction rates 


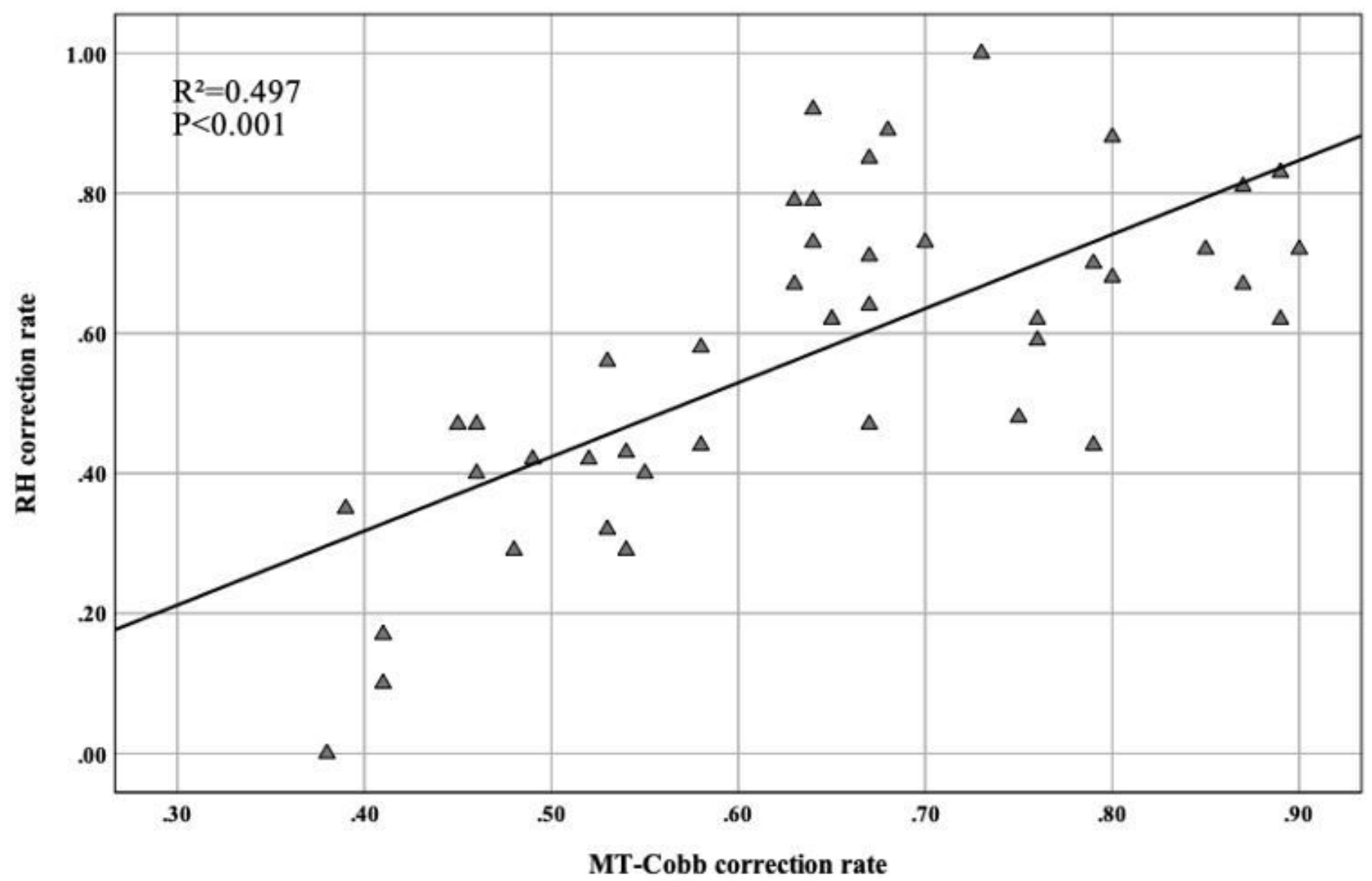

Figure 5

Correlation between MT-Cobb angle and apical rib hump prominence $(\mathrm{RH})$ correction rates 


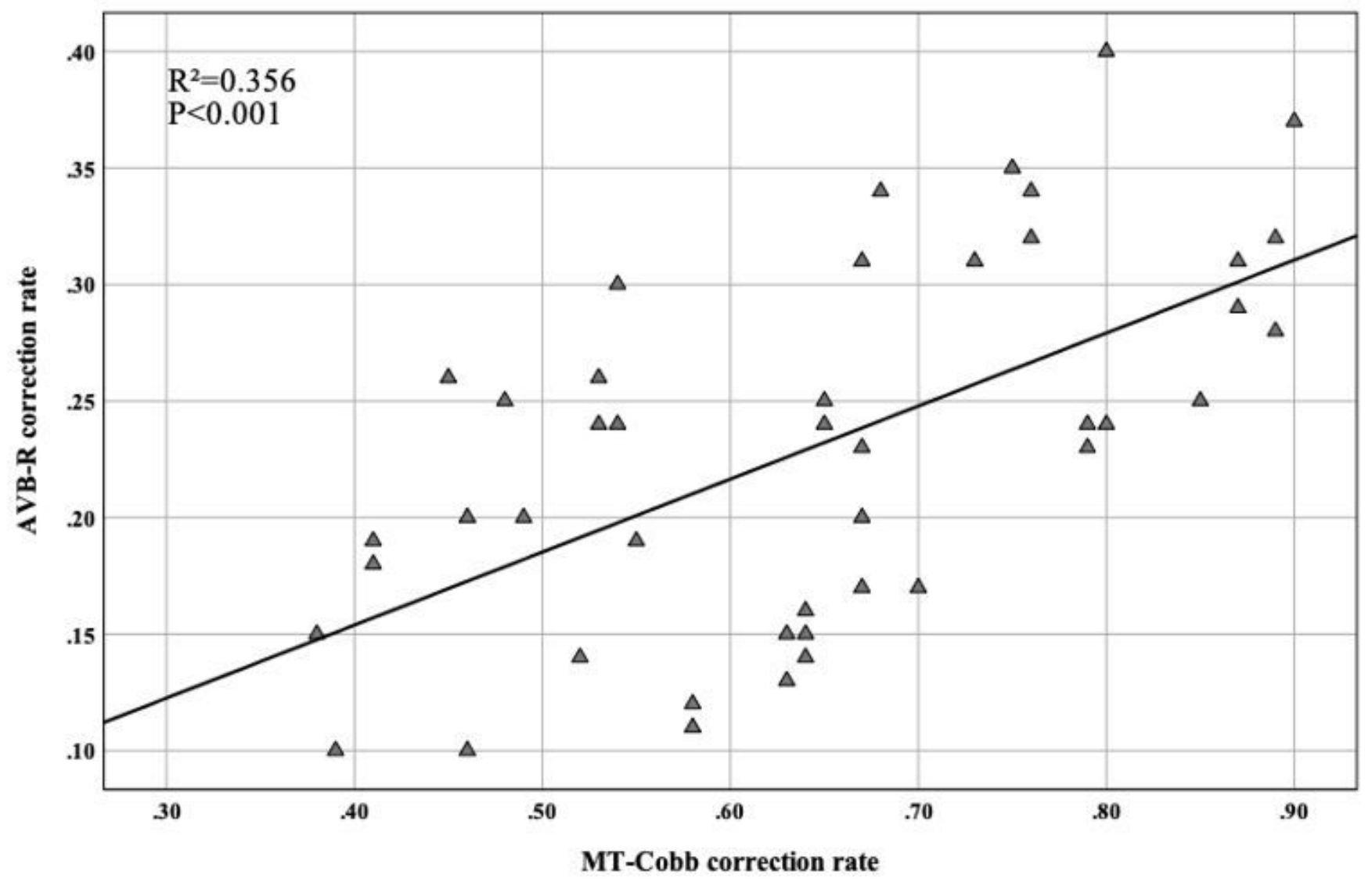

Figure 6

Correlation between MT-Cobb angle and apical vertebral body-rib ratio (AVB-R) correction rates 